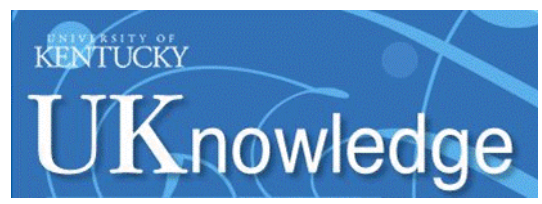

University of Kentucky

UKnowledge

$11-24-2020$

\title{
Hooked on a Feeling: Influence of Brief Exposure to Familiar Music on Feelings of Emotion in Individuals with Alzheimer's Disease
}

\author{
Alaine E. Reschke-Hernández \\ University of Kentucky, Alaine.ReschkeHernandez@uky.edu \\ Amy M. Belfi \\ Missouri University of Science and Technology \\ Edmarie Guzmán-Vélez \\ Massachusetts General Hospital \\ Daniel Tranel \\ University of lowa
}

Follow this and additional works at: https://uknowledge.uky.edu/music_facpub

Part of the Geriatrics Commons, and the Music Therapy Commons

Right click to open a feedback form in a new tab to let us know how this document benefits you.

\section{Repository Citation}

Reschke-Hernández, Alaine E.; Belfi, Amy M.; Guzmán-Vélez, Edmarie; and Tranel, Daniel, "Hooked on a Feeling: Influence of Brief Exposure to Familiar Music on Feelings of Emotion in Individuals with Alzheimer's Disease" (2020). Music Faculty Publications. 3.

https://uknowledge.uky.edu/music_facpub/3

This Article is brought to you for free and open access by the Music at UKnowledge. It has been accepted for inclusion in Music Faculty Publications by an authorized administrator of UKnowledge. For more information, please contact UKnowledge@lsv.uky.edu. 


\title{
Hooked on a Feeling: Influence of Brief Exposure to Familiar Music on Feelings of Emotion in Individuals with Alzheimer's Disease
}

\author{
Digital Object Identifier (DOI) \\ https://doi.org/10.3233/jad-200889 \\ Notes/Citation Information \\ Published in Journal of Alzheimer's Disease, v. 78, no. 3. \\ (C) 2020 IOS Press and the authors. All rights reserved.
}

The authors have purchased from the publisher the right to post this PDF file on their personal, their institute's, company's, or funding agency's Web site and in their repository. 


\title{
Hooked on a Feeling: Influence of Brief Exposure to Familiar Music on Feelings of Emotion in Individuals with Alzheimer's Disease
}

\author{
Alaine E. Reschke-Hernández ${ }^{\mathrm{a}, *}$, Amy M. Belfi $^{\mathrm{b}}$, Edmarie Guzmán-Vélez $^{\mathrm{c}}$ and Daniel Tranel ${ }^{\mathrm{d}, \mathrm{e}}$

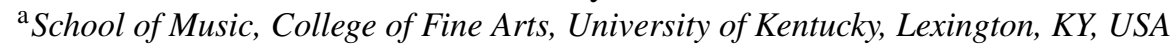 \\ ${ }^{\mathrm{b}}$ Department of Psychological Science, Missouri University of Science and Technology, Rolla, MO, USA \\ ${ }^{\mathrm{c}}$ Department of Psychiatry, Massachusetts General Hospital, Harvard Medical School, Boston, MA, USA \\ ${ }^{\mathrm{d}}$ Department of Psychological and Brain Sciences, University of Iowa, Iowa City, IA, USA \\ ${ }^{\mathrm{e}}$ Department of Neurology, Division of Neuropsychology and Cognitive Neuroscience, \\ Carver College of Medicine, University of Iowa, Iowa City, IA, USA
}

Handling Associate Editor: Amy Clements-Cortes

Accepted 11 September 2020

\begin{abstract}
.
Background: Research has indicated that individuals with Alzheimer's-type dementia (AD) can experience prolonged emotions, even when they cannot recall the eliciting event. Less is known about whether music can modify the emotional state of individuals with $\mathrm{AD}$ and whether emotions evoked by music linger in the absence of a declarative memory for the eliciting event.

Objective: We examined the effects of participant-selected recorded music on self-reported feelings of emotion in individuals with $\mathrm{AD}$, and whether these feelings persisted irrespective of declarative memory for the emotion-inducing stimuli.

Methods: Twenty participants with AD and 19 healthy comparisons (HCs) listened to two 4.5-minute blocks of self-selected music that aimed to induce either sadness or happiness. Participants reported their feelings at baseline and three times post-induction and completed recall and recognition tests for the music selections after each induction.

Results: Participants with AD had impaired memory for music selections compared to HCs. Both groups reported elevated sadness and negative affect after listening to sad music and increased happiness and positive affect after listening to happy music, relative to baseline. Sad/negative and happy/positive emotions endured up to 20 minutes post-induction.

Conclusion: Brief exposure to music can induce strong and lingering emotions in individuals with AD. These findings extend the intriguing phenomenon whereby lasting emotions can be prompted by stimuli that are not remembered declaratively. Our results underscore the utility of familiar music for inducing emotions in individuals with AD and may ultimately inform strategies for using music listening as a therapeutic tool with this population.
\end{abstract}

Keywords: Alzheimer's disease, dementia, feelings, emotion, memory, music

\footnotetext{
${ }^{*}$ Correspondence to: Alaine E. Reschke-Hernández, University of Kentucky, School of Music, 105 Fine Arts Building, Lexington, KY, 40506-0022, USA. Tel.: +1 859257 4536. E-mail: Alaine. ReschkeHernandez@uky.edu.
}

\section{INTRODUCTION}

Separating the experience of an emotion from the memory for what caused that emotion seems nearly impossible. However, prior studies have shown that 
emotions and memory for the eliciting event can be dissociated [1, 2]; that is, emotions can persist in the absence of the declarative memory for what caused the emotion. Such findings have important implications for how we interact with and care for individuals with Alzheimer's disease (AD), who display impaired memory for recent events early in the disease process.

In a study by Guzmán-Vélez and colleagues [2], individuals with $\mathrm{AD}$ rated their feelings before and three times after watching a set of films intended to induce either sadness or happiness. They also completed memory tests (free recall and recognition) for the films. Findings indicated that this protocol effectively induced the targeted emotions, and participants reported experiencing either sadness or happiness for up to 30 minutes post-induction-even when they had impaired declarative memory for the stimuli. Intriguingly, the less participants remembered about the films, the longer their sadness endured, an effect which might indicate difficulty with emotion regulation. A follow-up study showed that the ability to sustain an emotion is associated with amygdala volume, supporting the key role of the amygdala for regulating and sustaining an emotion [3]. Altogether, these findings indicate that the emotional state of individuals with dementia and other memory-related impairments can be greatly impacted by the actions of those around them (as we suggested in our initial study in patients with severe amnesia [1]), which has important implications for the treatment of persons with impaired memory. Here, we continued our investigation of this phenomenon by examining the impact of recorded familiar music on the emotional experiences of individuals with AD.

The influence of music on affective responses is well-documented [4-6]. Regardless of musical training, people often value music for its emotive qualities [5, 7-9]. Preferred music listening can promote pleasurable responses and engage neural reward systems (i.e., mesolimbic pathway [10-12]), and this is even true for individuals listening to self-selected sad music [13]. Research and anecdotal evidence show that music is important to persons with AD [14], and such individuals appear to respond favorably to music that is familiar and meaningful to them [15, 16]. The idea of using familiar music listening as a cost-effective therapeutic tool has gained visibility in recent years. Nonetheless, it remains to be established whether feelings of emotion that are induced by familiar music persist in individuals with $\mathrm{AD}$
[17-20], who are likely to quickly forget having listened to music or its properties (e.g., song, topic, artist [21]). A careful examination of the effects of familiar music on feelings of emotion in individuals with $\mathrm{AD}$ would contribute to our understanding of the lasting impact that music can have on the emotional state of an individual, in the context of impaired declarative memory. Such research could subsequently inform care strategies and the use of music listening as a therapeutic tool with this population.

In this study, we examined the effects of brief participant-selected recorded music on feelings of emotion in individuals with probable AD. We predicted that self-selected recorded music familiar to the participant would effectively induce emotions. We expected that participants would experience emotions consistent with the musical valence (i.e., positive emotions during positive music; negative emotions during negative music). We also predicted that participants would report prolonged feelings of the targeted emotion irrespective of declarative memory for the music itself.

\section{MATERIALS AND METHODS}

The Institutional Review Board at The University of Iowa approved this study, and we followed a written protocol to administer all procedures. All healthy comparison participants (HCs) provided written informed consent. Participants with probable AD provided written assent, and a legally authorized representative provided written informed consent.

\section{Participants}

Twenty participants with probable $\mathrm{AD}$ (10 women, 10 men) ages 64-89 volunteered for this study. We recruited these participants from the Benton Neuropsychology Clinic at the University of Iowa Hospitals and Clinics, the Alzheimer's Association Iowa Chapter, and via a University-wide email recruitment system. These participants had been previously diagnosed with $\mathrm{AD}$ by their primary care physician or a neurologist following the conventional McKhann et al. criteria [22]. We also recruited $19 \mathrm{HCs}$ (9 women, 10 men) through a University-wide email system and fliers posted at a senior center. HCs (ages 60-88) were matched to $\mathrm{AD}$ participants regarding sex, age, and educational level. To minimize factors that could interfere with our experimental procedures or con- 
found dependent variables of interest, we excluded candidates who did not speak or understand English fluently, were younger than 60 , had a neurological or intellectual disability (other than $\mathrm{AD}$ for the patient group), a psychiatric disorder (e.g., major depression or anxiety), history of alcohol or drug abuse, uncorrected severe vision impairment, or multiple behaviorally confounding central nervous system lesions.

\section{Instruments}

Because depression influences the intensity and duration of the experience of emotions and could complicate interpretation of the primary outcome of interest in our experiment (i.e., emotions), we screened all participants using the Beck Depression Inventory [23]. Participants also completed an adapted version of the Iowa Music Background Questionnaire [24] to assess lifelong music participation and training, attitudes about music, and listening habits (questions specific to changes in listening habits and music enjoyment before and after hearing loss and cochlear implantation were adapted or omitted). Most participants self-reported little to no formal training or music knowledge, but all found music very enjoyable and listened to music daily. Several participants in both groups informally participated in church choir. One participant with AD and one HC participant were professional musicians. We administered the Complex Ideational Material subtest of the Boston Diagnostic Aphasia Examination [25] to ensure participants with AD could understand basic written and spoken instructions. We evaluated the level of dementia severity for all participants with $\mathrm{AD}$ using the Clinical Dementia Rating scale [26]. Descriptive and neuropsychological data are displayed in Table 1.

\section{Emotion measure}

Following the same approach used in previous studies [1,2], we examined emotional state using selfreport rating scales. To keep this study as parallel as possible to previous work, these self-reports focused on happy and sad emotions (the participants' feelings), and we used the same emotion measure used in prior similar research (actual measure is published in [2]). The emotion measure consisted of three sections: 1) a 100-point visual analog scale (VAS) for happiness and sadness that ranged from "I don't feel sad/happy at all" (0) to "I feel extremely sad/happy" (100); 2) a 9-point bipolar valence scale that ranged from "extremely unpleasant" (0) to "extremely pleas-
Table 1

Demographic and neuropsychological data

\begin{tabular}{|c|c|c|}
\hline & \multicolumn{2}{|c|}{ M (SEM) } \\
\hline & $\operatorname{AD}(N=20)$ & $\mathrm{HC}(N=19)$ \\
\hline Age $(y)$ & $75.9(1.7)$ & $73.5(1.7)$ \\
\hline Education (y) & $16.2(0.8)$ & $17.1(0.7)$ \\
\hline Women:Men & 10:10 & $9: 10$ \\
\hline $\begin{array}{l}\text { Handedness } \\
\text { (Right:Left:Mixed) }\end{array}$ & $18: 2: 0$ & $18: 0: 1$ \\
\hline Beck Depression Inventory ${ }^{1}$ & $5.0(0.8)(n=13)$ & $2.9(0.6)$ \\
\hline \multicolumn{3}{|l|}{ Clinical Dementia Rating: ${ }^{2}$} \\
\hline Very Mild (0.5) & 10 & \\
\hline Mild (1) & 9 & \\
\hline Moderate (2) & 1 & \\
\hline $\begin{array}{l}\text { Complex Ideational Material }{ }^{3} \\
(n=18)\end{array}$ & $9.3(0.2)$ & \\
\hline Pure Tone Average $(\mathrm{dB}):^{4}$ & $(n=18)$ & \\
\hline Right Ear & $33.3(3.7)$ & $22.8(1.4)$ \\
\hline Left Ear & $31.8(3.5)$ & $23.9(1.4)$ \\
\hline
\end{tabular}

Values shown are mean (standard error of the mean) unless otherwise indicated. ${ }^{1}$ Scores range from 0 to 14 (minimal), 14 to 20 (mild), 20 to 29 (moderate), and $>29$ (severe) [23]; ${ }^{2}$ Scoring: 0 (unimpaired),.5 (very mild), 1 (mild), 2 (moderate), and 3 (severe impairment) [26]; ${ }^{3}$ This comprehension task (auditory, reading) ranges from 10 (full comprehension) to 0 (poor comprehension) [25]; ${ }^{4}$ Obtained by averaging hearing threshold (in decibels $\mathrm{dB}$ ) at 250,500,1000,2000, and $4000 \mathrm{~Hz}$; higher $\mathrm{dB}$ indicates louder volume; 20-40 dB PTA indicates mild hearing loss [29].

ant" (8); and 3) select emotion terms from the Differential Emotions Scale (DES) [27] (happy, sad, fear, anger, disgust, anxious, calm), which participants rated from "not at all/none" (0) to "extremely/a great deal" (8). During each induction block, participants rated their emotions using this measure at baseline, immediate post-induction, 10 minutes postinduction (after the memory test), and 20 minutes post-induction (see Fig. 1 for a timeline of the experiment). Each time they completed this measure, we repeated instructions and reminded participants to answer with regards to how they felt in the present moment, as honestly as possible, and that there were no right or wrong answers.

\section{Hearing screening}

Due to the age of our participants, many likely had some degree of hearing loss [28]. Thus, taking this into account was an important consideration in our research (where valid task participation relies on good functional hearing). Rather than exclude individuals on the basis of hearing loss or use of hearing aids, we asked all candidates if they enjoyed listening to music during the initial screening phone conversation (n.b. all who expressed interest in this study said they 


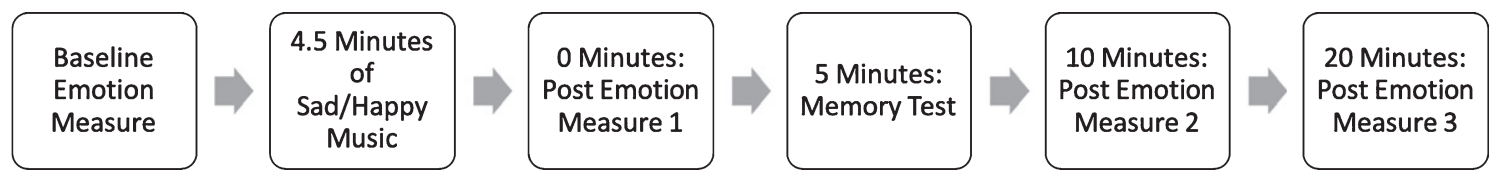

Fig. 1. Timeline depicting the within-subjects protocol for administering emotion measures, emotion induction procedures, and memory test. For ethical reasons, all participants completed the sad induction block followed by the happy induction block to help ensure they ended with a positive experience.

enjoyed listening to music). For descriptive purposes, we administered a pure tone audiometric screening following the modified Hughson-Westlake procedure [29] with a MAICO MA 25 manual air conduction audiometer to determine the lowest sound levels participants could hear at various frequencies. Briefly, in this protocol the experimenter begins at a decibel (dB) level that the participant can easily hear, then follows a "down 10/up 5" pattern to incrementally decrease the intensity $10 \mathrm{~dB}$. Once the participant no longer hears the tone, the experimenter increases the intensity $5 \mathrm{~dB}$. The final "up 5" $\mathrm{dB}$ level is recorded as the hearing threshold. We began at $60 \mathrm{~dB} 1000 \mathrm{Hertz}$ $(\mathrm{Hz})$ to check for understanding, and then obtained pure tone average $\mathrm{dB}$ hearing thresholds at 250, 500, 1000,2000 , and $4000 \mathrm{~Hz}$ in each ear. Two participants with $\mathrm{AD}$ declined to complete this hearing screening but were still included in the study. Hearing screening scores are displayed in Table 1.

\section{Music stimuli}

We opted to use participant-selected music, rather than researcher-selected music, as prior research suggests that music that participants know well and is personally meaningful is the most emotionally evocative $[4,5,9,10,12]$, and such familiar music is often the first-line choice for therapeutic music interventions with this population $[6,16,30]$. We asked participants to compile two ranked lists of familiar music (i.e., that they knew very well): 10 music selections that made them feel happy or positive and 10 that made them feel sad or negative. The experimenter emphasized that the selections must evoke emotions (as opposed to convey or represent emotions). Participants had up to a month to complete their lists, and we followed up weekly to inquire if they wanted assistance. Some participants with $\mathrm{AD}$ received help from a spouse or adult child; however, legally authorized representatives largely reported that these participants independently completed their lists. In some instances, participants could not generate a title or artist, so they hummed or sang the selection over the phone for Author 1 (a music therapist with extensive repertoire knowledge) who verified the selection with the participant. In other cases, participants generated 5-7 selections on each list easily and requested assistance with the final few selections. In such cases, Author 1 prompted, "have you thought of ..." and gave an example of a genre, era of music, or situation with which music is often paired.

All participants ranked their music selections from most- to least-evocative using a worksheet that we provided. We asked participants to indicate a musical artist for each selection, if that was important to them. If no artist was listed, we used the most popular recording of the selection from the participant's formative years [31]. Participants could also indicate if a segment of a selection was most emotive (e.g., beginning, middle, end; particular lyrics; minutes into the selection). We asked those who preferred longer selections to indicate the section that they found most emotive. For instance, one participant suggested Beethoven's 7th Symphony for their sad list, a 40-minute selection, and then indicated that the opening of the second movement evoked sadness.

We purchased all music selections from iTunes [32]. For each participant, we used clips from the first five selections on each of their music lists to create two individualized induction sets: one happy/positive and one sad/negative. Each music induction set lasted approximately 4.5 minutes (happy set average: 4 minutes 37 seconds; sad set average: 4 minutes 39 seconds). This duration is based on previous musicemotion induction research $[10,33,34]$ and takes into account the abbreviated attention span that is often observed in patients with $\mathrm{AD}$ [21]. We used Audacity version 2.1.2 [35] to create and edit these sets. Clips were arranged in reverse order, from least- to most-emotive according to the participants' rankings (i.e., selection 5 to 1 ), and we aimed to maintain the integrity of the musical phrase for each clip. All clips were presented in stereo, faded in and out, and were equalized to ensure volume was not erratic within a music set. A recorded windchime sounded between 
each music clip [36] (n.b. we inserted this attending sound to mimic the original protocol, in which a beep sounded between film clips [2]).

\section{Memory test}

We measured participants' recall and recognition of the music stimuli in a single instance 5 minutes post-induction, as indicated in Fig. 1. Memory recall was scored dichotomously (i.e., recalled/did not recall) and asked participants to name, recite lyrics, sing, and/or hum any music they remembered hearing in the past 10 minutes. We did not provide any additional prompts. Because participants had a wide range of musical training and background (novice to expert), we counted any response that indicated recall of a selection (Author 1 scored all responses). The recognition memory test comprised 15 brief clips (3-5 seconds each) that included a recognizable section of the selection (e.g., the chorus). Five clips were derived from the selections a participant listened to during the induction, 5 clips were on their list but not played during the induction (i.e., selections 6-10), and 5 were foils-similar in compositional style to the induction selections (e.g., Take the A Train by Duke Ellington foil for Little Brown Jug by Glenn Miller) or a different popular selection by the same artist (e.g., Credence Clearwater Revival, Bad Moon Rising foil for Down On the Corner). These clips faded in and out, were presented in a random order with 3 seconds of silence between clips, and participants responded yes or no regarding whether or not they heard the selection in the past 10 minutes. If a participant did not promptly respond, the researcher paused the music, and participants were offered an opportunity to listen to the clip a second time. We encouraged participants who remained unsure after a second opportunity to guess.

\section{Procedure}

Our procedures closely followed those described in previous similar research [1,2], the primary differences being the use of music and stimulus duration (18 minutes in previous studies; 4.5 minutes here). Briefly, the experiment consisted of two blocks: a sad block and a happy block. We wanted to prevent participants from leaving the lab in a negative mood without knowing why they felt that way; therefore, we purposefully used the same order for all participants: sad then happy. We followed the same procedures for both blocks, as illustrated in Fig. 1.
Participants rated their baseline feelings using the same emotion measure as in Guzmán-Vélez et al. (see Emotion measure section; [2]). Participants then set their preferred volume, bass, and treble levels using a Lepy LP-2020A hi-fi digital amplifier while listening to a 30-second music clip unrelated to the experiment [37]. In addition to the hearing screening previously described, this step helped ensure participants could hear the stimuli. To facilitate precise administration of the induction procedure, auditory stimuli (volume, baseline, induction set) automatically played from a CD using Windows Media Player on a PC computer via vintage stereo Baby Advent II speakers, which have a wide frequency response curve and offered more participant comfort and sensitivity to volume changes than headphones [38]. While we told participants that they would hear music, we did not inform them of the musical valence during the experiment.

Participants then listened to 4.5-minutes of selfselected music intended to induce sadness and rated their feelings when the music ended (see previous section Music stimuli). They completed a memory recall and recognition test 5 minutes after music listening. This test took about 5 to 10 minutes to complete (see Memory test section). Participants again rated their feelings approximately 10 to 15 minutes post-induction and finally at 20 to 25 minutes postinduction (time ranges reflect that some participants took longer to complete the memory test and emotion measures than others). During all waiting periods, we provided a written prompt to sit quietly on a computer monitor facing participants. We then took a 5-minute break before continuing with the happy induction block following these same procedures. We recorded behavioral observations throughout the experiment.

\section{Statistical analysis}

As in prior similar research [2], we converted all valence and DES emotion ratings (originally using scales of 0 to 8 ) into standardized units of $0 \%$ to $100 \%$, representing the "percent of maximum possible" (POMP) scores for each scale [39] before starting the statistical analysis. This conversion, after data collection, maintained the integrity of the psychometric properties of the measure and allowed us to analyze the valence and DES ratings on the same scale as the VAS ratings (which were scaled from 0 to 100 and thus already in POMP units). We then calculated composite scores for each induction to represent global changes in negative and positive affect. For the sadness induction, we calculated the nega- 

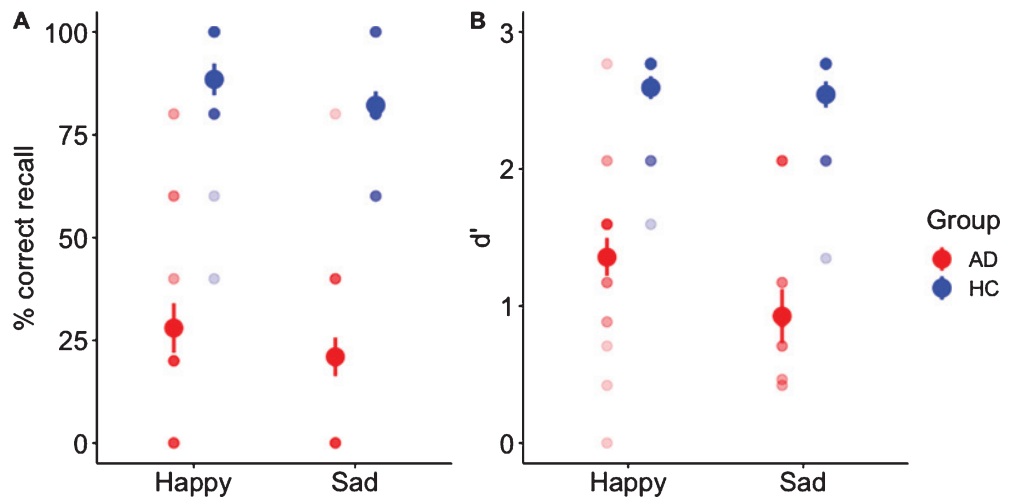

Fig. 2. Performance on memory tasks. Group means are denoted by the large opaque circles; individual subjects' datapoints are indicated by the transparent circles. A) Percent of selections that were recalled correctly. For both sad and happy emotion inductions, the HC group $(n=19)$ performed significantly better than the AD group $(n=20)$ on memory recall. B) Recognition memory (d', relative proportion of hits minus false alarms). For both sad and happy emotion inductions, the HC group performed significantly better than the AD group on memory recognition. Error bars indicate standard error of the mean.

tive affect composite score for each participant by averaging POMP scores for the following variables: sadness VAS; sadness, anger, disgust, fear, and anxiety from the DES; and valence (reverse scored). We calculated the positive affect composite score by averaging the happiness VAS, happiness DES, and calm DES scores.

For the happiness induction, we calculated the positive affect composite score for each participant by averaging the happiness VAS, happiness and calm DES, and valence scores. We calculated the negative affect composite score by averaging POMP scores for the following variables: sadness VAS and sadness, anger, disgust, fear, and anxiety DES scores.

We performed all statistical analyses using $R$ version 3.4.2 [40]. We calculated recall scores as the total number of selections correctly recalled divided by the total number of selections heard (5 each for happy and sad). For recognition memory, we calculated d' (hit rate - false alarm rate) to measure performance on the recognition test. As we did not predict an interaction between group and emotion induction, we conducted separate independent-samples $t$-tests to identify differences between the AD and $\mathrm{HC}$ groups on recall and recognition scores for each induction. Finally, we carried out a linear mixed-effects model for each emotion measure (VAS and POMP for positive and negative emotion) to identify differences in emotion ratings between the groups across time, as in prior work [41, 42]. To conduct these analyses, we used the lmer function from the lme 4 package [43] and the anova function from the car package to conduct significance tests [44]. These models included fixed effects for group, time, and their interaction, as well as random intercepts for participants.

\section{RESULTS}

\section{Sadness induction}

\section{Memory}

As expected, the AD group performed significantly lower than the HC group on both recall $(t(37)=-10.46, p<0.001,95 \%$ CI:[-0.72, -0.49$]$, $d=3.35)$ and recognition $(t(37)=-7.26, p<0.001$, 95\% CI: $[-2.06,-1.16], d=2.32$ ) for the sad music selections (see Fig. 2).

\section{Emotional state}

Overall, our results support our prediction that participants would experience emotions consistent with the sad music stimulus, regardless of declarative memory for the music itself. The sadness VAS data model revealed a significant main effect of time $[\mathrm{F}(3,111)=24.90, p<0.001]$, an interaction between time and group $[\mathrm{F}(3,111)=7.45, p<0.001]$, and a marginally-significant main effect of group $[F(1,37)=4.11, p=0.049]$. For the main effect of time, across groups, sadness VAS ratings were significantly above baseline at T1 (immediately after the emotion induction; $\beta=18.25, \mathrm{SE}=5.73, t=3.18$, $p=0.001$ ), T2 (after the memory test; $\beta=17.50$, $\mathrm{SE}=5.73, t=3.05, p=0.002$ ), and $\mathrm{T} 3$ (at the final rating; $\beta=13.00, \mathrm{SE}=5.73, t=2.26, p=0.025$ ). For 

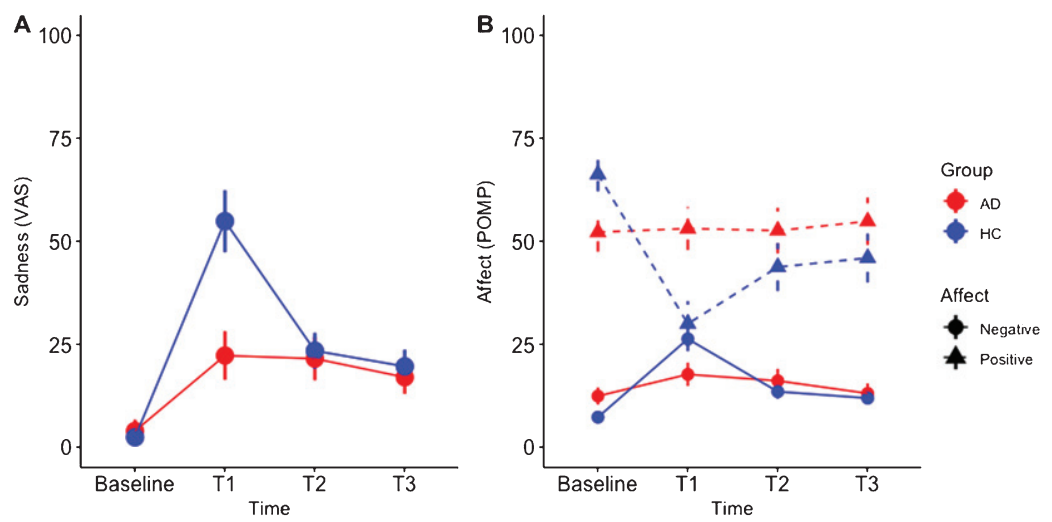

Fig. 3. Emotional responses of the AD group $(n=20)$ and the HC group $(n=19)$ to the sadness induction by music. A) Sadness VAS scores. Group means are denoted by the large opaque circles. For both groups, VAS ratings of sadness were significantly higher than baseline at all subsequent timepoints. B) Overall scores of positive and negative affect represented as a percent of maximum possible (POMP). Means are denoted by the large opaque circles (negative affect) and triangles (positive affect) for the AD group (red) and HC group (blue). Error bars indicate standard error of the mean.

the interaction between time and group, the $\mathrm{HC}$ group and the $\mathrm{AD}$ group significantly differed at T1 $(\beta=34.06, \mathrm{SE}=8.22, t=4.14, p<0.001)$. That is, while the $\mathrm{HC}$ group reported significantly higher feelings of sadness immediately after the emotion induction compared to the AD group, both groups reported similar levels of significantly elevated feelings at $\mathrm{T} 2$ and $\mathrm{T} 3$. Fig. $3 \mathrm{~A}$ presents a graphical depiction of these results.

We computed two additional models in response to the sadness induction: one for positive affect and one for negative affect. For positive affect in response to the sad music induction, there was a significant effect of time $[\mathrm{F}(3,111)=7.03, p<0.001]$, and a significant interaction between time and group $[\mathrm{F}(3,111)=7.77, p<0.001]$, but no significant main effect of group $[\mathrm{F}(1,37)=1.30, p=0.260]$. Compared to the AD group, the HC group reported significantly lower positive affect at $\mathrm{T} 1(\beta=-37.05$, $\mathrm{SE}=7.77, t=-4.76, p<0.001)$, T2 $(\beta=-22.82$, $\mathrm{SE}=7.77, t=-2.93, p=0.004)$, and T3 $(\beta=-22.87$, $\mathrm{SE}=7.77, t=-2.94, p=0.003)$ compared to baseline, while the $\mathrm{AD}$ group indicated no significant changes in positive affect in response to the sad music induction. For negative affect in response to sad music induction, there was a significant effect of time $[\mathrm{F}(3,111)=23.23, p<0.001]$ and a significant interaction between time and group $[\mathrm{F}(3,111)=7.72$, $p<0.001$, but no significant main effect of group $[F(1,37)=0.0008, p=0.977]$. Both groups showed a significant increase in negative affect at $\mathrm{T} 1(\beta=5.25$, $\mathrm{SE}=2.12, t=3.47 p=0.015$ ), although the HC group indicated a greater increase than the $\mathrm{AD}$ group at this timepoint $(\beta=13.68, \mathrm{SE}=3.04, t=4.48, p<0.001)$. See Fig. 3B for a graphical depiction of the data.

Seven participants with AD offer particularly compelling examples of this dissociation between their reported feelings and declarative memory for the eliciting event. At just 5 minutes post-induction, these participants could not recall any of their sad music selections, and 3 of them did not recall listening to music at all. Despite such severe memory impairment for music listening, participants reported sustained feelings of sadness up to 20 minutes post-induction. Fig. 4 illustrates these findings.

\section{Happiness induction}

\section{Memory}

In support of our hypothesis, the $\mathrm{AD}$ group performed significantly worse on recall $(t(37)=-8.32$, $p<0.001,95 \%$ CI: $[-1.56,-0.90], d=2.45)$ and recognition memory tests $(t(37)=-7.66, p<0.001$, 95\% CI:[-1.56, -0.90$], d=2.66)$ compared to the HC group for the happy music selections (see Fig. 2).

\section{Emotional state}

Our results for the happiness induction further support our prediction that participants would experience emotions consistent with the valence of the music, regardless of declarative memory for the music itself. As with the sad music results, the happiness VAS data model revealed a main effect of time $[\mathrm{F}(3,111)=11.56, p<0.001]$, but no main effect of group $[\mathrm{F}(1,37))=0.02, p=0.883]$ or interaction between time and group $[\mathrm{F}(3,111)=1.81, p=0.148]$. 

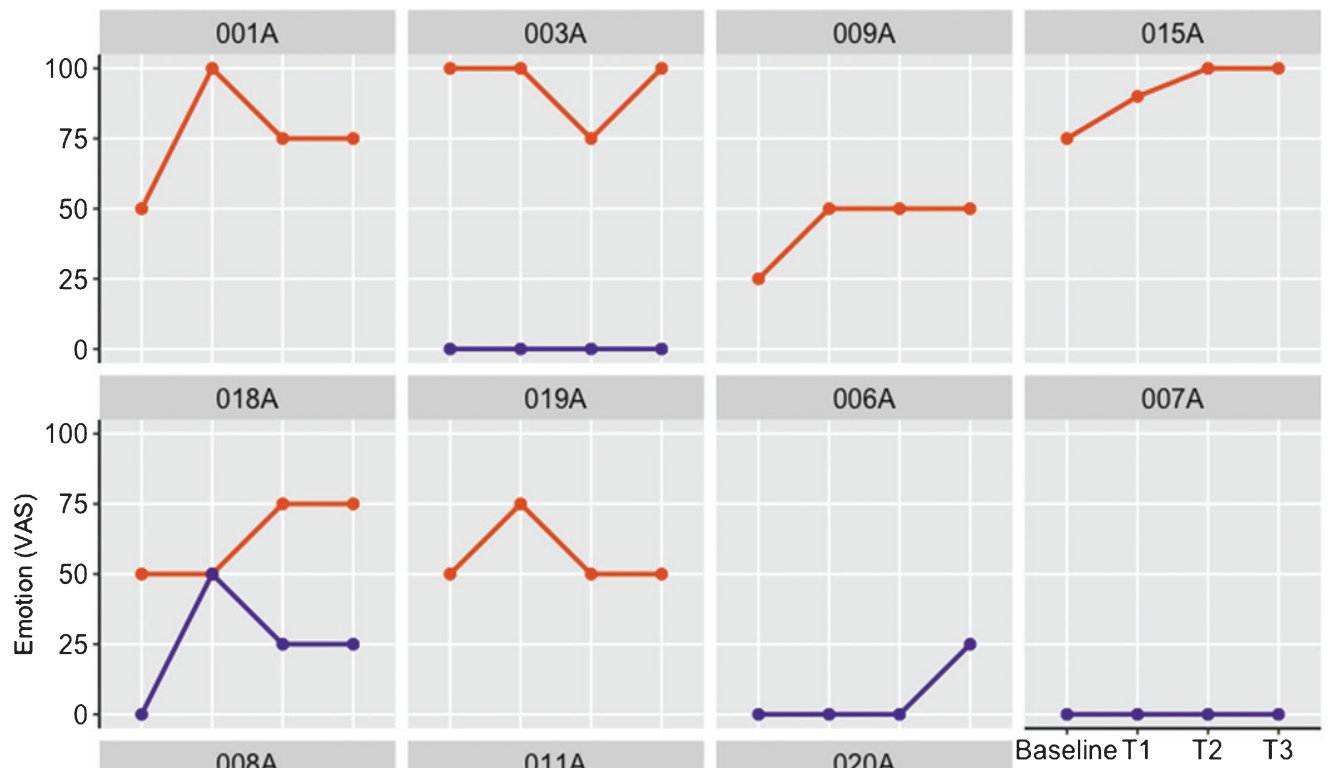

Subject

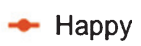

$\rightarrow$ Sad
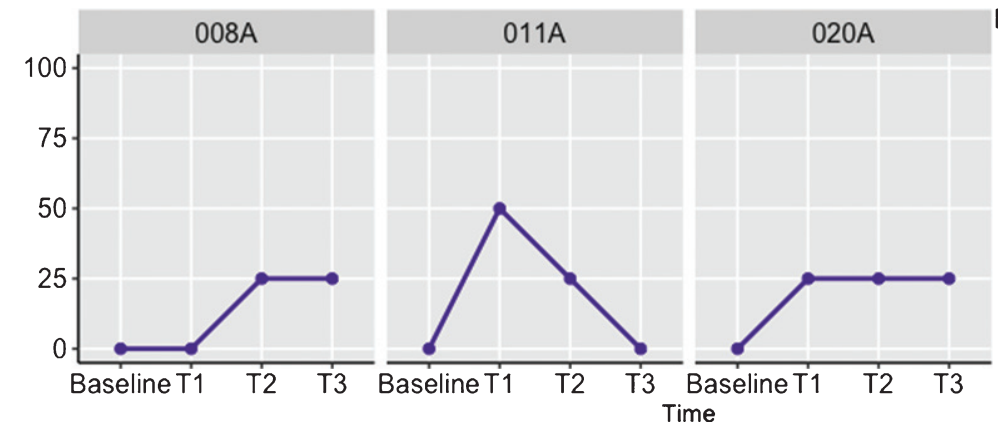

Fig. 4. Emotional responses to music in patients who demonstrated poor declarative memory for music that they had just heard during the experiment. Purple lines depict sadness ratings by 7 participants with $\mathrm{AD}$ who were unable to recall or recognize music selections that they had just heard during the sadness induction. Despite their severe memory impairment, 5 of these patients reported feeling a 25-50\% increase in sadness up to 20-minutes post-induction. Orange lines depict happiness ratings by 6 participants with AD who were unable to recall or recognize music selections that they had just heard during the happiness induction. Despite their severe memory impairment, 5 of these patients reported feeling a $25-50 \%$ increase in happiness up to 20 -minutes post-induction.
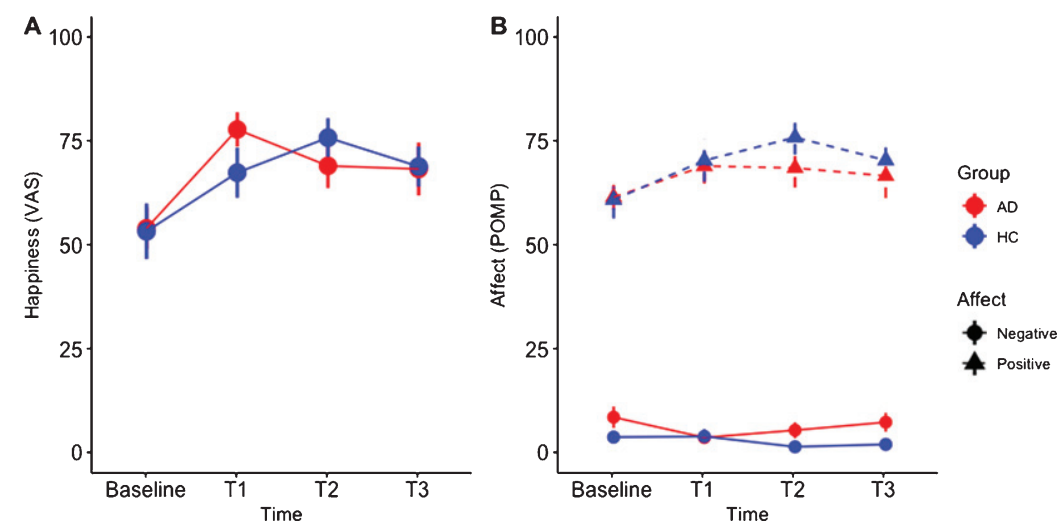

Fig. 5. Emotional responses of the AD group $(n=20)$ and the HC group $(n=19)$ to the happiness induction by music. A) Happiness VAS scores. Group means are denoted by the large opaque circles. For both groups, VAS ratings of happiness were significantly higher than baseline at all timepoints. B) Overall scores of positive and negative affect represented as a percent of maximum possible (POMP) [39]. Means are denoted by the large opaque circles (negative affect) and triangles (positive affect) for the AD group (red) and HC group (blue). Error bars indicate standard error of the mean. 
That is, happiness VAS ratings were significantly higher than baseline at $\mathrm{T} 1 \quad(\beta=23.75, \mathrm{SE}=5.20$, $t=4.56, p<0.001), \mathrm{T} 2(\beta=15.00, \mathrm{SE}=5.20, t=2.88$, $p=0.004)$, and $\mathrm{T} 3(\beta=14.25, \mathrm{SE}=5.20, t=2.73$, $p=0.007$ ) for both groups. Figure 5A offers a graphical depiction of these results.

For positive affect in response to the happy music induction, there was a significant effect of time $[\mathrm{F}(3,111)=5.93, p<0.001]$, but no significant main effect of group $[\mathrm{F}(1,37)=0.30, p=0.585]$ or interaction between time and group $[\mathrm{F}(3,111)=0.78$, $p=0.505]$. Across both groups, there was a marginally significant increase in positive affect at $\mathrm{T} 1$ $(\beta=7.50, \mathrm{SE}=3.83, t=1.95, p=0.052)$. For negative affect in response to happy music induction, there was no significant main effect of time $[\mathrm{F}(3,111)=2.06$, $p=0.109]$ or group $[\mathrm{F}(1,37)=3.29, p=0.077]$, and no significant interaction between group and time $[\mathrm{F}(3,111)=2.27, p=0.083]$. Figure $5 \mathrm{~B}$ provides a graphical depiction of these results.

Similar to the sadness induction, six participants with $\mathrm{AD}$ could not recall any of their happy music selections. Of these participants, 1 did not recall listening to music at all. Despite severe memory impairment for music listening, participants reported sustained feelings of happiness up to 20 minutes postinduction. Figure 4 illustrates these findings.

\section{DISCUSSION}

The aim of this study was to examine the effects of music on feelings of emotion in individuals with $\mathrm{AD}$, and to determine whether these feelings persisted irrespective of declarative memory for the emotioninducing stimuli. Using music that was familiar to participants as the stimulus, our findings contribute to the understanding of how music-evoked emotions can be affected in individuals with AD. Both the $\mathrm{AD}$ and $\mathrm{HC}$ participant groups reported feeling significantly sadder or happier after they listened to self-selected music. Further, participants with AD reported feeling sad or happy for at least 20 minutes beyond music listening despite their impaired declarative memory for the music stimuli that triggered these feelings. Our findings have two key implications. First, familiar music can effectively induce positive and negative emotions in individuals with AD. Second, how we treat and interact with individuals with memory impairment can have a lasting impact on their emotional state, regardless of whether they recall what happened to them.

\section{Emotions linger in individuals with $A D$}

A dissociation between memories and emotions induced by music is most striking when participants have a strong, lasting emotional response to music stimuli, while at the same time having impaired declarative memory for the music itself following the induction. This is precisely what we found. Participants with $\mathrm{AD}$ had impaired (in some cases, zero) recollection and recognition for the stimuli five minutes after listening to music. Yet, they reported experiencing lasting sadness or happiness. These findings are consistent with those from Feinstein et al. [1] and Guzmán-Vélez and colleagues [2, 3], who showed that individuals with amnesia caused by hippocampal damage could experience lasting emotions induced by films. Our findings provide evidence that familiar music listening can induce lingering emotions in individuals with $\mathrm{AD}$, and that the affective experience associated with music can occur and persist independently from the declarative memory for that music.

\section{Distinctions from past research}

Important differences between the current study and previous studies in this line of research should be noted. Results of both Guzmán-Vélez et al. [2] and Feinstein et al. [1] indicated a stronger effect of the sad films on emotions compared to happy films. By contrast, in the current study, happy and sad familiar music listening protocols induced similar degrees of emotional response. One explanation could relate to differences in induction duration-participants viewed 18 minutes of film clips compared to just 4.5 minutes of music listening in our study. However, stimulus duration does not explain the different responses to happy and sad films relative to happy and sad music. A more plausible explanation could relate to the media itself. In the film induction research, most of the happy film clips depicted amusing situations and were largely verbal and/or image-based (i.e., jokes, funny home videos). By contrast, sad clips largely derived from movies and included music. Among its various functions, film music is composed to direct attention, communicate meaning, and convey and evoke emotion. Composers exploit a variety of techniques to ensure the music is evocative [45]. It seems the multidimensional aspects of stimuli used in past research likely contributed to findings. 


\section{How did music evoke emotions?}

Past research has indicated the importance of using participant-selected music to elicit strong emotions [10-12]. Familiar music, particularly music from the formative years of late adolescence and early adulthood, often evokes autobiographical memories and associated emotions [46-48]. As one participant with AD stated, "(I feel this way because) this is great music from my youth and happy times." In our study, both $\mathrm{AD}$ and $\mathrm{HC}$ participants provided personally meaningful, multifarious music lists from all manner of music genres, including popular, folk, contemporary Christian, showtunes, hymns, rock-nroll, patriotic, big band, jazz, classical, and country. A preliminary examination of the music selected by participants with $\mathrm{AD}$ indicates that much of it was derived from their formative years, whereas HCs seemed to include more of a mix of selections from across their lifespan. This observation indicates a possible bias regarding remote music-evoked autobiographical memories (MEAMs) in individuals with AD. Indeed, some research suggests that familiar music may play a greater role in prompting the recall of self-defining memories for individuals with dementia than it does for healthy older adults [15]. Thus, it seems likely that MEAMs contributed in part to the changes in emotional state experienced by the participants in our study.

Our data also suggest a somewhat different pattern of response in the $\mathrm{AD}$ group compared to the HC group. Specifically, while the HC group showed increases in negative affect and decreases in positive affect in response to sad music, the AD group demonstrated increased negative affect and stable positive affect. Prior work has indicated that experiencing such mixed emotions when listening to music is associated with feelings of nostalgia in young, healthy participants [49], suggesting that individuals with AD may have experienced greater nostalgia after listening to their selections. However, this phenomenon is yet to be fully examined in a sample of older adults.

Selections that elicited emotions did not per se align with compositional features that often prompt categorization of music as happy or sad (i.e., music in major mode with a faster tempo is often labeled happy, whereas minor mode and slower tempo music is often labeled sad [50]). As an illustrative example, Sweet Caroline (Neil Diamond) is a relatively fast tempo selection in major mode with a driving beat and a catchy, repetitious chorus. While some participants included this song on their happy list, for one HC participant, listening to just seconds of this selection evoked a self-reported decrease in positive affect and happiness with concomitant increase in negative affect and sadness and overt behavioral response (crying, grimacing, slumped posture). This participant listed this song as their most sad selection and disclosed that it was associated with a sad memory. To further illustrate the inherent difficulty in selecting music to evoke a particular emotional response, certain selections, such as Amazing Grace and Hey Jude (The Beatles) appeared at the top of some participants' happy lists, and at the top of others' sad lists. This is not surprising-it is the very nature of music.

While MEAMs and nostalgia may have been involved, such associations are one of at least eight putative routes by which music can evoke emotion, including rhythmic entrainment, music-evoked visual imagery, and musical expectancy, among others [5, 7-9]. It is likely that the music-induced emotions seen here arose from a combination of such factors $[5,9]$. The goal of our study was to effectively induce either sadness or happiness irrespective of whether or not an autobiographical memory, or some other variable, mediated the process. More research is needed to elucidate which mechanisms most strongly influence emotional responses to music in this population. Also, research should systematically examine these mechanisms in racially and ethnically diverse samples of both healthy and cognitively impaired older adults to fully understand music-emotion evocation phenomena and their relevance to music interventions. Nevertheless, the present work takes the important initial step of indicating that familiar music does induce strong emotions in persons with AD.

\section{Ethical considerations for music listening interventions}

In addition to positive responses, our results indicate that familiar music listening could likewise evoke negative affective responses, as in prior similar research $[1,2]$. When asked why they felt the way they did, participants in our study who had no recall for sad music stated, "I think my dog died," "I'm just not... I haven't done enough for myself and I get mad at myself," "I am worried about the future," and "Sometime, something happened to me. I just don't know." Given the emotional evocativeness of music, an important consideration 
is the extent to which individuals with significant memory impairment can effectively self-regulate and cope with emotions evoked by experiences they may not remember. For instance, caregivers have described exacerbated behavioral and psychological symptoms in response to music listening [51]. Past research has further indicated that the manner of music listening (i.e., with headphones) may cause overstimulation or enhance social isolation for patients with dementia [16, 52]. As recently suggested within the music-induced harm model [53], future studies could examine possible short- and long-term adverse side effects as well as benefits of music listening to improve our understanding of the best use and appropriate implementation of musicbased interventions with persons with dementia and other cognitively impaired populations. Furthermore, given these considerations regarding music selection and potential for adverse responses, the characteristics of the recipient as well as the expertise and resources of the person implementing a music listening protocol within the care milieu should be taken into account $[30,53]$.

\section{Limitations and future directions}

Our study had several limitations. Based on our stated aims and prior research [10-12], we used participant-selected familiar music to maximize the likelihood of inducing the targeted emotions. Although we took steps to maximize reproducibility and enhance the rigor of our experiment through written protocols, this choice sacrificed a degree of experimental control (viz., different participants listened to different music). Choice and arrangement of music stimuli, from familiar participant-selected to highly controlled sets of music, are important and necessary considerations in future research to fully understand underlying mechanisms of affective responses to music in persons with $\mathrm{AD}$ and older adults more generally.

As in past research, we chose not to counterbalance the order of presentation of happy and sad induction blocks (for ethical reasons), and this may have influenced our results. Our sample included a near equal number of men and women, but it was homogeneous with regards to ethnicity and educational attainment. People who expressed interest in participating may have had particular affinity for music, and as such, our results may not be representative of the larger population of persons with AD. Future studies could include a more diverse sample to expand our knowledge regarding this phenomenon and inform culturally sensitive music intervention practices.

To facilitate comprehension of the self-report measure and other aspects of our experiment, we included participants with relatively mild $\mathrm{AD}$ and tested their comprehension before they completed the experiment. Although we informally screened healthy participants (see Participants section), we did not formally test these participants' comprehension or cognition. As in any study involving human participants, there is a chance that some did not understand an aspect of our experiment. While our inclusion criteria and design of our experiment helped maximize participant comprehension and testing stamina, future studies could examine the impact of music on emotions in individuals at more advanced stages of the disease as well as with other neurodegenerative diseases (e.g., frontotemporal dementia). Finally, we did not examine the contribution of various mechanisms to music-evoked emotions in our experiment. Future research could examine the degree to which MEAMs and other such mechanisms contribute to emotions induced by music in individuals with dementia and the role affective responses play in therapeutic music interventions.

While much is known regarding healthy and younger individuals' responses to music [4, 5, 9], systematic investigation regarding the impact of music on emotional states in older adults and individuals with cognitive impairment is much more limited [7, $8,30]$. Our findings contribute foundational evidence regarding familiar music's impact on emotions in older adults with memory impairment. Such research pertaining to the influence of music on non-musical responses informs the theoretical and scientific foundation for music therapy and related interventions [54-56]. While the results of our study prompt considerations regarding responses to music listening with this population, additional research concerning the valence, intensity, and duration of affective responses (i.e., feelings, emotions, and longer-term mood and psychosocial wellbeing) and their reliable measurement in those with more advanced stages of $\mathrm{AD}$ and related dementias is warranted (see for example [30]). Examining the possible mechanisms involved to better understand how music-based interventions work and to inform best practices with this population is another research priority.

This study broadens our understanding of emotional responses of individuals with $\mathrm{AD}$, specifically with regards to familiar music. Results underscore the importance of considering emotions in the gen- 
eral care of persons with memory impairment and can be used to inform the implementation of therapeutic music interventions. In general, studies regarding music and emotion tend to focus on young, healthy participants (i.e., college undergraduates). Such findings may not apply to older adults per se. Future studies could build on our current work to examine music and emotion phenomena in various healthy and clinical older adult populations, including those with $\mathrm{AD}$. As the number of individuals with $\mathrm{AD}$ and other dementias increases, it is imperative that we identify tools that can help improve the psychosocial wellbeing of both the patients and their caregivers. Our findings suggest that familiar music can profoundly impact the emotions of both healthy older adults and individuals with $\mathrm{AD}$ and may ultimately help clarify mechanisms underlying therapeutic applications of music with this population.

\section{ACKNOWLEDGMENTS}

The authors thank all the participants and their care partners, without whom this research would not have been possible, and the Alzheimer's Association Iowa Chapter for assistance with recruitment. The authors thank Mónica Acevedo-Molina, Kuan-Hua Chen, Kathy Jones, Jasmine Leahy, Kenneth Manzel, Ashwathi Nair, Meghan Ross, Sawyer Small, Morgane Tatchoum, Tiffany Theulen, and Keara Turkington for their assistance on this project; Nicholas Jones for his technical assistance; Virginia Driscoll for her guidance on audiometric screening in older adults; and Joel Bruss for his input on stereo music system setup and speaker donation. This work was supported by the Iowa State Fraternal Order of Eagles and the Kiwanis Neuroscience Research Foundation.

Authors' disclosures available online (https://www. j-alz.com/manuscript-disclosures/20-0889r1).

\section{REFERENCES}

[1] Feinstein JS, Duff MC, Tranel D (2010) Sustained experience of emotion after loss of memory in patients with amnesia. Proc Natl Acad Sci U S A 107, 7674-7679.

[2] Guzmán-Vélez E, Feinstein J, Tranel D (2014) Feelings without memory in Alzheimer disease. Cogn Behav Neurol 27, 117-129.

[3] Guzmán-Vélez E, Warren DE, Feinstein JS, Bruss J, Tranel D (2016) Dissociable contributions of amygdala and hippocampus to emotion and memory in patients with Alzheimer's disease. Hippocampus 26, 727-738.

[4] Koelsch S (2010) Towards a neural basis of music-evoked emotions. Trends Cogn Sci 14, 131-137.
[5] Juslin PN (2013) From everyday emotions to aesthetic emotions: Toward a unified theory of musical emotions. Phys Life Rev 10, 235-266.

[6] Gfeller KE (2008) Music: A human phenomenon and therapeutic tool. In An Introduction to Music Therapy: Theory and Practice, Davis WB, Gfeller KE, Thaut MH, eds. American Music Therapy Association, Silver Spring, MD, pp. 41-75.

[7] Laukka P (2007) Uses of music and psychological wellbeing among the elderly. J Happiness Stud 8, 215-241.

[8] Saarikallio S (2010) Music as emotional self-regulation throughout adulthood. Psychol Music 39, 307-327.

[9] Hargreaves D, North A (2011) Experimental aesthetics and liking for music. In Handbook of Music and Emotions: Theory, Research, Applications, Juslin PN, Sloboda JA, eds. Oxford University Press, New York, pp. 515-546.

[10] Blood A, Zatorre RJ (2001) Intensely pleasurable responses to music correlate with activity in brain regions implicated in reward and emotion. Proc Natl Acad Sci U S A 98, 1181811823.

[11] Ferreri L, Mas-Herrero E, Zatorre RJ, Ripollés P, GomezAndres A, Alicart H, Olivé G, Marco-Pallarés J, Antonijoan RM, Valle M, Riba J, Rodriguez-Fornells A (2019) Dopamine modulates the reward experiences elicited by music. Proc Natl Acad Sci U S A 116, 3793-3798.

[12] Pereira CS, Teixeira J, Figueiredo P, Xavier J, Castro SL, Brattico E (2011) Music and emotions in the brain: Familiarity matters. PLoS One 6, e27241.

[13] Vuoskoski JK, Eerola T (2017) The pleasure evoked by sad music is mediated by feelings of being moved. Front Psychol 8, 439.

[14] McDermott O, Orrell M, Ridder HM (2014) The importance of music for people with dementia: The perspectives of people with dementia, family carers, staff and music therapists. Aging Ment Health 18, 706-716.

[15] El Haj M, Antoine P, Nandrino JL, Gely-Nargeot M-C, Raffard S (2015) Self-defining memories during exposure to music in Alzheimer's disease. Int Psychogeriatr 27, 17191730.

[16] Gerdner L (1998) The effects of individualized vs classical "relaxation" music on the frequency of agitation in elderly persons with Alzheimer's disease and related disorders, The University of Iowa (Doctoral dissertation).

[17] Baird A, Samson S (2015) Music and dementia. In Music, Neurology, and Neuroscience, Altenmüller E, Finger S, Boller F, eds. Elsevier, Waltham, MA, pp. 207-235.

[18] Raglio A, Bellandi D, Baiardi P, Gianotti M, Ubezio MC, Zanacchi E, Granieri E, Imbriani M, Stramba-Badiale M (2015) Effect of active music therapy and individualized listening to music on dementia: A multicenter randomized controlled trial. J Am Geriatr Soc 63, 1534-1539.

[19] Raglio A, Pavlic E, Bellandi D (2018) Music listening for people living with dementia. J Am Med Dir Assoc 19, $722-723$.

[20] van der Steen JT, Smaling HJA, van der Wouden JC, Bruinsma MS, Scholten R, Vink AC (2018) Musicbased therapeutic interventions for people with dementia. Cochrane Database Syst Rev 7, CD003477.

[21] Anderson SW, Cassell MD (2015) Alzheimer's disease. In Neuropsychology of Cortical Dementias, Noggle C, Dean R, Bush SS, eds. Springer, New York, pp. 93-120.

[22] McKhann GM, Knopman DS, Chertkow H, Hyman BT, Jack CR, Kawas CH, Klunk WE, Koroshetz WJ, Manly JJ, Mayeux R, Mohs RC, Morris JC, Rossor MN, Scheltens P, Carrillo MC, Thies B, Weintraub S, Phelps $\mathrm{CH}$ 
(2011) The diagnosis of dementia due to Alzheimer's disease: Recommendations from the National Institute on Aging-Alzheimer's Association workgroups on diagnostic guidelines for Alzheimer's disease. Alzheimers Dement 7, 263-269.

[23] Beck AT, Steer RA, Carbin MG (1988) Psychometric properties of the Beck Depression Inventory: Twenty-five years of evaluation. Clin Psychol Rev 8, 77-100.

[24] Gfeller K, Christ A, Knutson J, Witt S (2000) Musical backgrounds, listening habits, and aesthetic enjoyment of adult cochlear implant recipients. $J$ Am Acad Audiol 11, 390-406.

[25] Goodglass H(2001) The Assessment of Aphasia and Related Disorders, Lippincott, Williams, \& Wilkins, Philadelphia.

[26] Morris JC (1993) The Clinical Dementia Rating (CDR): Current version and scoring rules. Neurology 43, 24122414.

[27] Izard CE, Dougherty FE, Bloxom BM, Kotsch NE (1974) The Differential Emotions Scale: A Method of Measuring the Meaning of Subjective Experience of Discrete Emotions, Vanderbilt University, Department of Psychology, Nashville.

[28] National Institute on Deafness and Other Communication Disorders (2013) Hearing Loss and Older Adults, U.S. Department of Health and Human Services, National Institutes of Health, Bethesda, MD.

[29] Carhart R, Jerger JF (1959) Preferred methods for clinical determination of pure-tone thresholds. J Speech Lang Hear Res 24, 330-345.

[30] Reschke-Hernández AE (2019) A clinical practice model of music therapy to address psychosocial functioning for persons with dementia: Model development and randomized clinical crossover trial, University of Iowa (Doctoral dissertation), https://doi.org/10.17077/etd.59oh-y06y.

[31] Gibbons AC (1977) Popular music preferences of elderly people. J Music Ther 14, 180-189.

[32] Apple Inc. (2000-2019) iTunes.

[33] Fairclough SH, van der Zwaag M, Spiridon E, Westerink J (2014) Effects of mood induction via music on cardiovascular measures of negative emotion during simulated driving. Physiol Behav 129, 173-180.

[34] Hilz MJ, Stadler P, Gryc T, Nath J, Habib-Romstoeck L, Stemper B, Buechner S, Wong S, Koehn J (2014) Music induces different cardiac autonomic arousal effects in young and older persons. Auton Neurosci 183, 83-93.

[35] Audacity Team (1999-2018) Audacity.

[36] Redfern L, Daydreaming, Accessed at https://soundbible. com/2030-Daydreaming.html on July 23, 2020.

[37] MacLeod K, Morning Snowflake, Accessed at https://www. freepd.com on July 23, 2020.

[38] Burack OR, Jefferson P, Libow LS (2003) Individualized music: A route to improving the quality of life for long-term care residents. Act Adapt Aging 27, 63-76.

[39] Cohen P, Cohen J, Aiken LS, West SG (1999) The problem of units and the circumstance for POMP. Multivar Behav Res 34, 315-346.
[40] R Core Team. (2018) R: A Language and Environment for Statistical Computing. R Foundation for Statistical Computing, https://www.R-project.org.

[41] Belfi AM, Kasdan A, Rowland J, Vessel EA, Starr GG, Poeppel D (2018) Rapid timing of musical aesthetic judgments. J Exp Psychol Gen 147, 1531-1543.

[42] Belfi AM, Vessel EA, Starr GG (2018) Individual ratings of vividness predict aesthetic appeal in poetry. Psychol Aesthet Creat Arts 12, 341-350.

[43] Bates D, Mächler M, Bolker B, Walker S (2015) Fitting linear mixed-effects models using lme4. J Stat Softw 67, $1-48$.

[44] Fox J, Weisberg S (2011) An R Companion to Applied Regression, Sage, Thousand Oaks, CA.

[45] Cohen AJ (2010) Music as a source of emotion in film. In Handbook of Music and Emotions: Theory, Research, Applications, Juslin PN, Sloboda JA, eds. Oxford University Press, New York, pp. 879-908.

[46] Janata P, Tomic ST, Rakowski SK (2007) Characterisation of music-evoked autobiographical memories. Memory 15, 845-860.

[47] Schulkind MD, Hennis LK, Rubin DC (1999) Music, emotion, and autobiographical memory: They're playing your song. Mem Cognit 27, 948-955.

[48] Belfi AM, Karlan B, Tranel D (2015) Music evokes vivid autobiographical memories. Memory 24, 979-989.

[49] Barrett F, Grimm K, Robins R, Wildschut T, Sedikides C, Janata P (2010) Music-evoked nostalgia: Affect, memory, and personality. Emotion 10, 390-403.

[50] Vieillard S, Peretz I, Gosselin N, Khalfa S, Gagnon L, Bouchard B (2008) Happy, sad, scary and peaceful musical excerpts for research on emotions. Cogn Emot 22, 720-752.

[51] Robinson L, May C, Bond J, Hutchings D, Corner L, Beyer F, Dickinson H, Vanoli A, Finch T, Hughes J, Ballard C (2006) A systematic literature review of the effectiveness of non-pharmacological interventions to prevent wandering in dementia and evaluation of the ethical implications and acceptability of their use. Health Technol Assess 10, iii, ix-108.

[52] Clair AA, Bernstein B (1995) The effect of no music, stimulative background music and sedative background music on agitated behaviors in persons with severe dementia. Act Adapt Aging 19, 61-70.

[53] Silverman MJ, Gooding LF, Yinger O (2020) It's... complicated: A theoretical model of music-induced harm. J Music Ther 57, 251-281.

[54] Hanson-Abromeit D (2015) A conceptual methodology to define the therapeutic function of music. Music Ther Perspect 33, 25-38.

[55] Thaut MH (2000) The scientific model (R-SMM). In A Scientific Model of Music in Therapy and Medicine. IMR Press, San Antonio, TX, pp. 1-24.

[56] de L'Etoile SK, Dachinger C, Fairfield J, Lathroum L (2012) The Rational-Scientific Mediating Model (R-SMM): A framework for scientific research in music therapy. Music Ther Perspect 30, 130-140. 